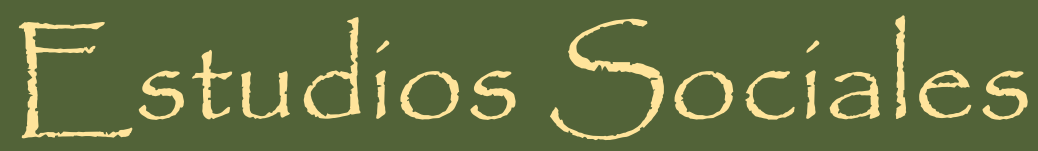

Revista de Alimentación Contemporánea y Desarrollo Regional

Volumen 29, Número 54. Julio - Diciembre 2019

Revista Electrónica. ISSN: 2395-9169

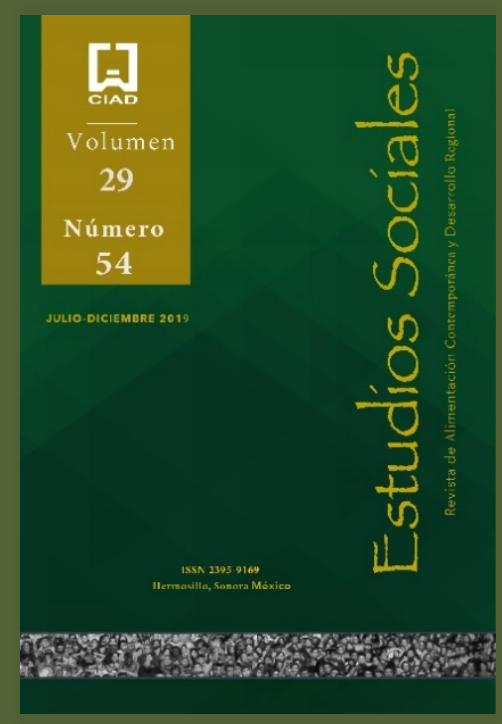

Evolución de la política de apoyo

a la alimentación en las alternancias políticas

y el cambio en la situación alimentaria

en las entidades federativas mexicanas entre 2010 y 2015

Food support policy evolution

in political alternations and change

in the alimentary situation

in the Mexican federal entities between 2010 and 2015

DOI: https://dx.doi.org/10.24836/es.v29i54.825

PII: e19825

María Abril Becerra-Montes*

https://orcid.org/0000-0001-7733-6133

Gerardo Ordóñez-Barba*

https://orcid.org/0000-0003-3498-5808

Fecha de recepción: 22 de mayo de 2019.

Fecha de envío a evaluación: 14 de agosto de 2019.

Fecha de aceptación: 27 de agosto de 2019.

*El Colegio de la Frontera Norte, México.

Autor para correspondencia:

Gerardo Ordóñez-Barba.

Departamento de estudios de Administración Pública.

Carretera escénica Tijuana-Ensenada, Km. 18.5.

San Antonio del Mar, 22560.

Tijuana, B. C., México.

Teléfono (664) 631-6300 ext. 5316

Dirección electrónica: ordonez@colef.mx

Centro de Investigación en Alimentación y Desarrollo, A. C.

Hermosillo, Sonora, México.

(c) (1) (9) 


\section{EVOLUCIÓN DE LA POLÍTICA DE APOYO A LA ALIMENTACIÓN EN LAS ALTERNANCIAS POLÍTICAS Y EL CAMBIO EN LA SITUACIÓN ALIMENTARIA \\ EN LAS ENTIDADES FEDERATIVAS MEXICANAS ENTRE 2010 Y 2015}

BECERrA-MONTES, ORdÓNEEZ-BARBA

\section{Resumen / Abstract}

Objetivo: Analizar la política alimentaria mexicana durante el periodo 2000-2018; cuantificar los niveles de la intervención de cuatro programas de apoyo al consumo de alimentos y los cambios en la carencia alimentaria a nivel estatal entre 2010 y 2015, y explorar el posible efecto de la intervención de tales programas sobre la carencia alimentaria. Metodología: Análisis documental y estadístico a partir de fuentes secundarias; este último incluyó la construcción de indicadores y la aplicación de la técnica de correlación estadística para cuantificar el grado de relación entre los niveles de cobertura y gasto de los cuatro programas en estudio a escala estatal, así como entre éstos y el comportamiento de la carencia alimentaria. Resultados: Los programas no comparten un patrón de distribución de recursos específico, aunque todos muestran una expansión de su cobertura relacionado con el aumento en su nivel de gasto. No obstante, el incremento de la intervención del conjunto de programas observado entre 2010 y 2015, de acuerdo con el análisis exploratorio realizado, no tuvo un efecto significativo en la reducción de la carencia y, en algunos estados, no logró evitar su incremento entre ambos años. Limitaciones: La parte del estudio exploratorio no profundiza en los factores contextuales que pudieran estar condicionando los resultados de los programas, para lo cual se requieren de nuevas investigaciones que delimiten su capacidad de intervención ante cambios importantes del entorno económico o social. Conclusiones: Los resultados indican que debe cuestionarse la efectividad de los recursos asignados a ciertos programas alimentarios, así como los criterios bajo los cuales se determinan entidades federativas prioritarias.

Palabras clave: alimentación contemporánea; política alimentaria; seguridad alimentaria; programas sociales; situación alimentaria, intervención estatal.
Objective: Analyze the Mexican food policy in the period 2000-2018; measure the intensity of the intervention of four food programs and the changes in the levels of food shortage at state level between 2010 and 2015 and the effect of those programs on the lack of food in the Mexican states. Methodology: Documentary and statistical analysis from secondary sources; the latter included the construction of indicators and the application of the statistical regression technique to quantify the relationship between coverage levels and spending of four food support programs at the state level, as well as between these and the behavior of food shortage between 2010 and 2015. Results: The programs do not share a specific resources distribution pattern, although all show an expansion of their coverage, probably related to the increase in their spending level. However, according to the analysis carried out, the increase in the intervention of the set of programs between 2010 and 2015, did not have a significant effect in the reduction of the food shortage and, in some states, it did not avoid its increase between both years. Limitations: The exploratory study section does not delve into the contextual factors that could be conditioning the results of the programs, for which new research is required that limits the intervention capacity of the programs in the face of important changes in the economic or social environment. Conclusions: The results show that it should be questioned the effectiveness of the resources assigned to certain food programs, as well as the criteria under which the priority federal entities are determined.

Key words: contemporary food; food policy; food security; social programs; food situation; state intervention. 


\section{Introducción}

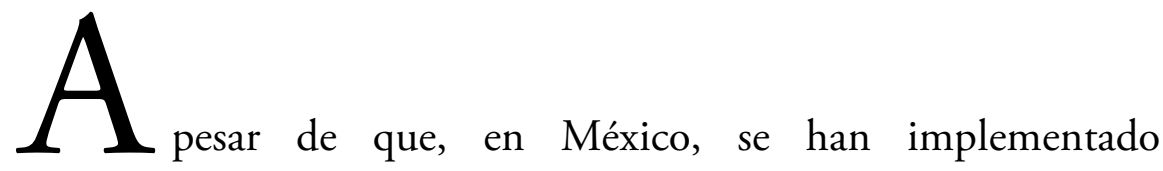
programas sociales de apoyo a la alimentación desde hace varias décadas, hoy en día, una quinta parte de la población nacional sigue enfrentando carencia en el acceso a una alimentación adecuada (Coneval, 2018). De acuerdo con Ordóńez (2002) y Huerta (2005), las reformas promovidas, a partir de la década de los ochenta del siglo pasado, se han traducido en un cambio importante en la conceptualización de las políticas públicas y, por ende, en las políticas sociales promovidas por el Estado. En esta época se optó por reconfigurar la política alimentaria federal hacia el otorgamiento de apoyos solo a los grupos vulnerables a fin de mejorar su alimentación mediante programas focalizados. Debido a la constante implementación de programas alimentarios y a que, actualmente, sigue habiendo millones de mexicanos que padecen esta carencia, autores como Barquera, Rivera-Dommarco y Gasca-García (2001), Cardozo (2005) y Coneval (2011a) han cuestionado su eficiencia y eficacia.

En su análisis sobre la política alimentaria, el Consejo Nacional de Evaluación de la Política de Desarrollo Social (Coneval), haciendo referencia a los Programas Oportunidades, de Apoyo Alimentario (PAL), de Abasto Rural (PAR) y de Abasto Social de Leche (PASL), ha señalado que 
a pesar del incremento presupuestario en varios de los programas, éstos no pudieron contener el incremento en la carencia de acceso a la alimentación entre 2008 y 2010. Si bien los programas han tenido algunos resultados respecto al acceso a la alimentación, el efecto negativo de la reducción del ingreso y el aumento en el precio de los alimentos que el país sufrió desde 2007 excedió el efecto de los programas sociales aquí analizados (Coneval, 2011a, p. $31)$.

Atender el problema de la falta de una alimentación sana y suficiente resulta imperativo, puesto que una persona que no recibe una alimentación adecuada a sus necesidades está en riesgo de desarrollar problemas de salud que le dificulten vivir su vida de manera plena. La importancia de la alimentación es tal que es concebida como un derecho social, tanto por organismos nacionales como internacionales y, por tanto, es deber del Estado asegurar que todas las personas tengan acceso a una alimentación suficiente y nutritiva. En este contexto, el presente trabajo tiene tres objetivos principales: el primero consiste en analizar la evolución que han experimentado tanto la política alimentaria mexicana como los programas de apoyo al consumo de alimentos a partir del año 2000, cuando se produce la primera alternancia política en el gobierno federal; el segundo objetivo es cuantificar la intensidad de la intervención de cuatro programas alimentarios y medir los cambios en los niveles de carencia alimentaria a nivel estatal entre 2010 y 2015; finalmente, un tercer propósito consiste en explorar el posible efecto de la intervención de estos programas sobre el cambio en la carencia alimentaria en las entidades federativas durante el periodo en estudio.

El artículo está compuesto por cuatro apartados y las conclusiones. En el primero se presenta una revisión del recorrido de la política alimentaria mexicana a partir del 2000 y a lo largo de tres sexenios, incluyendo los cambios que se vislumbran con el inicio de la nueva administración 2018-2024. En el segundo apartado se describen las características generales de los programas alimentarios que hasta el 2018 seguían vigentes en el presupuesto nacional, poniendo especial atención en los programas sociales que facilitan el acceso a la alimentación de los mexicanos. De estos últimos, en el tercer apartado se cuantifica la intensidad de la intervención de cuatro programas a nivel estatal, considerando los indicadores de gasto y cobertura que registraron entre 2010 y 2015 y, para el mismo periodo, se mide el cambio en los niveles de carencia alimentaria observados en las 32 
entidades federativas. Enseguida, en el cuarto apartado, se presentan los resultados del posible efecto de la intervención de los programas estudiados en el apartado anterior sobre el comportamiento de la carencia alimentaria a nivel estatal. Finalmente, en las conclusiones se presentan algunas reflexiones acordes con los resultados obtenidos.

\section{La política alimentaria en los gobiernos de las alternancias políticas, 2000- 2018}

La política social mexicana se modificó sustancialmente después de las reformas estructurales impuestas en la década de los ochenta del siglo pasado, produciendo un cambio importante en la conceptualización de las políticas, que a su vez supuso una reconfiguración de los programas sociales. Lo anterior dio lugar a que los programas con objetivos alimentarios se volvieran mayormente focalizados y se volcaran hacia nuevas modalidades de apoyos, como las Transferencias Monetarias Condicionadas (TMC) y los apoyos dirigidos hacia grupos vulnerables (mujeres embarazadas, nińos en edad escolar, adultos mayores, etc.), eliminando así los subsidios generalizados que habían sido el principal medio de apoyo a la población en general.

Siguiendo las directrices marcadas por los gobiernos anteriores, desde el año 2000 la política social mexicana ha tenido como uno de sus principales ejes de actuación la atención a la pobreza, sobre todo, extrema, a partir de esfuerzos como el incremento en la cobertura del Programa de Educación Salud y Alimentación (Progresa), creado en 1997, cuyo método de intervención se realiza a través de las llamadas Transferencias Monetarias Condicionadas (TMC). ${ }^{1}$ Además, se han establecido diferentes estrategias (Contigo es posible, Vivir mejor, Cruzada Nacional contra el Hambre) en el marco de cada administración federal con el objetivo de superar la pobreza a través de otro conjunto de acciones y programas focalizados en arenas de política pública diversas como el empleo (Programa de Empleo Temporal), la vivienda (Vivah, Hábitat), la salud (Seguro Popular) o la seguridad social (60 y más), entre otros.

En el ámbito alimentario, el primer gobierno surgido de la alternancia política a nivel federal hizo poco para modificar los patrones de actuación heredados, es 
decir, dio continuidad al proceso de reestructuración de la política que se inició durante el gobierno de Miguel de la Madrid (1982-1988), mediante la cual se propuso modificar las dimensiones de la intervención estatal en todas las fases del ciclo alimentario (producción, transformación, comercialización y consumo), de tal forma que pudieran beneficiar solo a la población más pobre y atender a un estricto control de prioridades (Ordóńez, 2017). Como lo apuntan BrachetMárquez y Sherraden,

en la práctica se conservarían los subsidios al consumo de tortilla y leche, pero convertidos en programas restringidos que alcanzaban a los pobres de manera irregular y azarosa, debido a los complejos procedimientos burocráticos para lograr los documentos necesarios para obtener esos beneficios (BrachetMárquez y Sherraden, 1993, p. 345).

Esta reorientación fue el núcleo de las directrices adoptadas por las tres administraciones que siguieron en turno (incluyendo la del Presidente Fox Quezada, 2000-2006), mediante la cual se desmanteló la Conasupo hasta su extinción en 1999 y se aplicaron los subsidios específicos. Ante la situación de escasez e incremento de los precios internacionales de los alimentos básicos, el gobierno del Presidente Felipe Calderón (2006-2012) puso en marcha en 2008 una estrategia emergente denominada Acciones de Apoyo a la Economía Familiar con tres objetivos principales: facilitar el abasto y el acceso de los consumidores a los mejores precios de los alimentos en el mercado internacional, impulsar la producción de alimentos y aumentar la productividad en el campo, y proteger el ingreso y fortalecer la economía de las familias más pobres (Presidencia de la República, 2008). Entre las acciones que se desarrollaron se encontraban: la reducción o eliminación de aranceles a la importación de trigo, arroz, maíz, frijol, leche en polvo, sorgo, pasta de soya, fertilizantes nitrogenados e insumos químicos; el fortalecimiento de todos los programas que apoyan a los productores de maíz, frijol, caña de azúcar y leche; el sostener sin cambios el precio de la leche Liconsa y la harina de maíz que se vende en las tiendas Diconsa; y, por último, incrementar los montos de dinero en 120 pesos mensuales que se otorgaban a beneficiarios de Oportunidades y de los programas alimentarios para Zonas Marginadas y de Diconsa (Ordóñez, 2017). 
Sólo unas cuantas de las acciones anteriores se mantuvieron hasta el final del sexenio del Presidente Felipe Calderón y muy pocas lograron trascender al gobierno del Presidente Enrique Peña Nieto, quien depositó el principal esfuerzo en materia alimentaria en la llamada Cruzada Nacional Contra el Hambre $(\mathrm{CNCH})$, que en realidad se trató de "una estrategia cuya intervención se basa en la coordinación de programas o acciones entre distintas instancias (y niveles) gubernamentales en torno a espacios territoriales específicos" (Yaschine, Ochoa, y Hernández, 2016, p. 425). Desde que se anunció su constitución fue objeto de múltiples críticas, tanto por las inconsistencias de su diseño y el desfase que presenta con las dimensiones de la seguridad alimentaria que promueve la Organización de las Naciones Unidas para la Alimentación y la Agricultura (FAO) y los objetivos del Reto Hambre Cero de la ONU (Yaschine, Ochoa y Hernández, 2016), como por la manera tan restrictiva en que define y cuantifica a su población objetivo (Boltvinik, 2016). En opinión de Barba (2016), la $\mathrm{CNCH}$ se caracteriza por su falta de transparencia y claridad. Además, este autor cuestiona la presencia de representantes de empresas fabricantes de alimentos bajos en nutrientes como PepsiCo y Nestlé, así como la subestimación de la población objetivo respecto de las estimaciones de Coneval (2018).

Considerando su diseńo, es claro que los efectos sociales que pudiera generar la $\mathrm{CNCH}$ dependían de los logros de una multiplicidad de programas federales (principalmente de Prospera, el Seguro Popular y el Programa de Apoyo Alimentario), así como del empeño que los gobiernos locales pusieran en el proyecto. La única iniciativa que fue concebida para apoyar directamente a la Cruzada fue el Programa de Comedores Comunitarios, cuyos beneficios parecen insuficientes para resolver el problema alimentario en el país (Ordóñez, 2017).

En el gobierno de López Obrador, que apenas inició en diciembre de 2018, ya se vislumbran cambios en torno a la política alimentaria del país, cuyos principales objetivos son atender la situación de hambre, alcanzar la seguridad alimentaria, mejorar la nutrición, así como promover la agricultura sustentable (Sánchez, 2018). En este sentido, en 2019 se conformó un nuevo organismo llamado Seguridad Alimentaria Mexicana (Segalmex), ${ }^{2}$ encargado de promover la seguridad alimentaria del país mediante el acopio de productos básicos, el establecimiento de precios de garantía, la promoción de la industrialización de productos, entre otras medidas (SADER, 2019). 
Así, de acuerdo con López y Sandoval (2019), la administración de López Obrador ha optado por centrar el combate a la pobreza y desnutrición mediante el fortalecimiento del campo y la producción de alimentos básicos, por lo que consideran que la actual administración es más ambiciosa que la anterior en cuanto al logro de la seguridad alimentaria. Entre las acciones implementadas en 2019 destaca el aumento de productos incluidos en la canasta básica, que pasó de 23 a 40 (SADER, 2019b). Adicionalmente, se ha sustituido a Prospera por el Programa Beca Universal para Estudiantes de Educación Media Superior Benito Juárez, el cual será implementado por la Secretaría de Educación Pública (SEP) y se limita a entregar dinero para evitar la deserción escolar, dejando de ofrecer apoyos para salud y alimentación (DOF, 2019).

Del análisis de la evolución de la política alimentaria mexicana se puede concluir que ha ido transformándose de una manera desarticulada y con una alta propensión hacia la focalización cada vez más extrema. Como veremos en el apartado siguiente con más detalle, los programas que se han venido incorporando a lo largo de varias décadas y que seguían operando hasta 2018, han dado lugar a una política fragmentada y carente de integralidad, que dificulta la convergencia y la eficacia de los esfuerzos emprendidos.

Por otro lado, es importante dar seguimiento a las acciones que el actual gobierno federal está emprendiendo en un esfuerzo por reestablecer precios de garantía, que ya en la década de los sesenta se emplearon mediante el Comité Regulador de Mercados de Subsistencia (Ceimsa), posteriormente, conocida como Compañía Nacional de Subsistencias Populares (Conasupo). Cabe destacar que el encargado de la Segalmex fue titular de Conasupo entre 1988 y 1990. En este contexto, resultará particularmente interesante analizar si el regreso de acciones emprendidas décadas atrás en periodos económicos y sociales sumamente diferentes a los actuales pueden generar mejores resultados a los obtenidos recientemente, los cuales han sido poco alentadores. 
ESTUDIOS SOCIALES

ISSN: 2395-9169

\section{Programas federales con objetivos alimentarios en México}

El Coneval lleva a cabo la medición del acceso a la alimentación a partir de la Escala Mexicana de Seguridad Alimentaria (EMSA), la cual permite determinar si un hogar tiene seguridad alimentaria o si, por el contrario, presenta algún grado de inseguridad. Esta conceptualización fue formulada por la Organización de las Naciones Unidas para la Alimentación y la Agricultura (FAO) y su definición se ha adoptado por convención internacional.

De acuerdo con este organismo, "existe seguridad alimentaria cuando todas las personas tienen, en todo momento, acceso económico a suficientes alimentos, inocuos y nutritivos para satisfacer sus necesidades alimenticias y sus preferencias en cuanto a los alimentos, a fin de llevar una vida activa y sana" (FAO, 1996). Para dar viabilidad a este concepto, la FAO lo divide en tres dimensiones: la oferta adecuada de alimentos durante todo el año a nivel nacional y local (disponibilidad); el acceso físico y económico de los hogares a alimentos que sean de buena calidad y en cantidades suficientes (acceso) y que la preparación de los alimentos asegure que las necesidades de los integrantes de las familias queden satisfechas (utilización biológica). En México los programas sociales con objetivos alimentarios contemplan entre sus premisas el concepto de seguridad alimentaria (MD CONSULTORÍA, 2012).

A fin de ofrecer una idea del tipo de acciones e iniciativas que componen la política alimentaria que regía en el país, en el Cuadro 1 se presenta el listado de programas con objetivos alimentarios implementados por el gobierno federal hasta el 2018. Dicho concentrado fue realizado con base en una revisión de diversos artículos académicos y de reportes elaborados por Coneval (2009, 2011a y 2011b), a partir de lo cual se propone una clasificación de los programas según la dimensión de la seguridad alimentaria a la que contribuyen. Es importante puntualizar que el presente trabajo gira en torno al estudio de algunos programas alimentarios que, mayormente, contribuyen a mejorar el acceso a la alimentación y de los cuales se ofrecerá una revisión más detallada posteriormente. 


\section{EVOLUCIÓN DE LA POLÍTICA DE APOYO A LA ALIMENTACIÓN EN LAS ALTERNANCIAS POLÍTICAS \\ Y EL CAMBIO EN LA SITUACIÓN ALIMENTARIA \\ EN LAS ENTIDADES FEDERATIVAS MEXICANAS ENTRE 2010 Y 2015}

BECERra-MONTES, ORdóNEEZ-BARBA

\section{Cuadro 1.}

\section{Programas federales con objetivos alimentarios implementados en México, 2018}

\begin{tabular}{|c|c|}
\hline Estrategia o programa & Dependencia encargada \\
\hline Programa de Abasto Social de Leche (PASL) & LICONSA S.A de C.V. \\
\hline Programa de Abasto Rural (PAR) & DICONSA S.A. de C.V. \\
\hline Estrategia Integral de Asistencia Social Alimentaria (EIASA) & Sistema Nacional DIF (SNDIF) \\
\hline PROSPERA Programa de Inclusión Social** & SEDESOL* \\
\hline Programa de Apoyo Alimentario (PAL) & DICONSA S.A. de C.V. \\
\hline Programa de Apoyo Alimentario en Zonas de Atención Prioritaria (PAAZAP) & SEDESOL* \\
\hline Cruzada Nacional Contra el Hambre & SEDESOL* \\
\hline Programa de Comedores Comunitarios & SEDESOL* \\
\hline Programa de Albergues Escolares Indígenas & CDI \\
\hline \multicolumn{2}{|l|}{ Disponibilidad } \\
\hline Apoyo a la Cadena Productiva de los Productores de Maíz y Frijol (PROMAF) & Sagarpa \\
\hline Proyecto Estratégico para la Seguridad Alimentaria (PESA) & $\begin{array}{l}\text { SNDIF y Sist. Estatales DIF } \\
\text { (SEDIF) }\end{array}$ \\
\hline Programa de Comercialización y Desarrollo de Mercados & SAGARPA \\
\hline $\begin{array}{l}\text { Programa de Desarrollo de Capacidades, Innovación Tecnológica y } \\
\text { Extensionismo Rural }\end{array}$ & SAGARPA \\
\hline Programa de Fomento a la Agricultura & SAGARPA \\
\hline Programa de Fomento a la Productividad Pesquera y Acuícola & SAGARPA \\
\hline Programa de Fomento Ganadero & SAGARPA \\
\hline Programa de Sanidad e Inocuidad Agroalimentaria & SAGARPA \\
\hline Programa Integral de Desarrollo Rural & SAGARPA \\
\hline Programa de Productividad y Competitividad Agroalimentaria & SAGARPA \\
\hline PROAGRO Productivo & SAGARPA \\
\hline Programa MAS AGRO & SAGARPA/CIMMYT \\
\hline Programa de Inducción y Desarrollo del Financiamiento del Medio Rural & SAGARPA \\
\hline Apoyo a la Inversión y Equipamiento en Infraestructura & CONAPESCA \\
\hline Fondo de Estabilización para la Comercialización de la Leche & SAGARPA \\
\hline Programa de Prevención y Manejo de Riesgos & SAGARPA \\
\hline Adquisición de Activos Productivos & SAGARPA \\
\hline Uso Sustentable de Recursos Naturales para la Producción Primaria & SAGARPA \\
\hline \multicolumn{2}{|l|}{ Utilización biológica } \\
\hline Estrategia Integral de Asistencia Social Alimentaria (EIASA) & $\begin{array}{l}\text { SNDIF/ Secretaría de Salud } \\
\text { (SALUD) }\end{array}$ \\
\hline Estrategia Nacional para la Prevención del Sobrepeso, la Obesidad y la Diabetes & SEDESOL* \\
\hline Comunidad DIFerente & SNDIF \\
\hline Programa de Entornos y Comunidades Saludables & SALUD \\
\hline
\end{tabular}

Fuente: elaboración propia con base en Coneval (2009, 2011a), Sistema Nacional DIF (2010), Ávila, Flores y Rangel (2011), Ordóńez (2012), Franco y Canela (2016). *A partir del 1 de diciembre de 2018 se llama Secretaría de Bienestar (BIENESTAR). 
De la revisión de los programas con objetivos alimentarios se identificaron un total de 29, así como dos estrategias de intervención que son implementados por distintas dependencias federales entre las que destacan Sedesol y Sagarpa. Aunque los programas tienen objetivos diferentes entre sí, todos buscan coadyuvar al logro de la seguridad alimentaria. Para el año 2018 había 18 programas relacionados con la dimensión de disponibilidad, de los cuales uno depende de la Comisión Nacional de Acuacultura y Pesca (Conapesca) y resto de la Sagarpa. Dichos programas buscan fortalecer la producción de alimentos a través de regulaciones del mercado y del impulso a la producción de alimentos (maíz, frijol, leche) y actividades diversas (pesqueras, acuícolas, ganaderas, agrícolas). Respecto a los programas alineados con la utilización biológica, se identificaron cuatro que en general buscan promover una alimentación sana de acuerdo con las necesidades de los individuos. Dichos programas son implementados por el SNDIF, así como por la Secretaría de Salud y Sedesol.

Respecto a la dimensión de acceso, al 2018 había siete programas y dos estrategias vinculadas. El más antiguo, el Programa de Abasto Social de Leche (PASL), fue creado en 1961 y su objetivo es contribuir a que la población con ingresos por debajo de la Línea de Bienestar Mínimo (LBM, que equivale a un ingreso por persona inferior al valor de la canasta alimentaria) consuma leche fortificada. En orden cronológico le sigue el PAR, creado en 1979, cuya misión ha sido facilitar el acceso físico y económico a alimentos en localidades de alta y muy alta marginación, mediante el abasto de tiendas rurales donde se ofertan productos a precios inferiores a los de mercado.

En 1997 se pone en marcha Progresa, el primer programa de Transferencias Monetarias Condicionadas (TMC) en el país, cuyo propósito, aunque se ha mantenido en esencia, ha cambiado con el tiempo como producto de las mutaciones que sufrió en 2002, cuando cambio su nombre a Oportunidades, y en 2015, cuando se transformó en Prospera (véase Ordóńez y Silva, 2019). Ya en esta última etapa se estableció como su objetivo

contribuir a fortalecer el cumplimiento efectivo de los derechos sociales que potencien las capacidades de las personas en situación de pobreza a través de acciones que amplían el desarrollo de sus capacidades en alimentación, salud y educación y el acceso a otras dimensiones de bienestar para coadyuvar a la ruptura del ciclo intergeneracional de la pobreza (Sedesol, 2014). 
En 2001 inició la implementación de la Estrategia Integral de Asistencia Social Alimentaria (EIASA) a cargo del Sistema Nacional para el Desarrollo Integral de la Familia (SNDIF), que incluye a cuatro programas sociales a través de los cuales se busca contribuir a la seguridad alimentaria de diferentes grupos poblacionales. A partir de 2003 se implementa el Programa de Apoyo Alimentario (PAL), que contribuye a mejorar la alimentación de sus beneficiarios mediante apoyos económicos.

Desde 2008 entró en operación el Programa de Apoyo Alimentario en Zonas de Atención Prioritaria (PAAZAP), el cual brinda nutrición y alimentación a integrantes de hogares de localidades marginadas que no son atendidos por otros programas. A partir de 2009, este programa se fusionó con el PAL (Coneval, 2009) y ambos pasaron a formar parte de la estructura del Prospera en 2016, aunque como veremos enseguida con el PAL se siguen reportando cifras de forma separada.

Por último, en 2013 inició la Cruzada Nacional Contra el Hambre (CNCH), estrategia que buscaba erradicar las causas de la pobreza extrema y de la inseguridad alimentaria a partir de las acciones de 70 programas sociales pertenecientes a 19 dependencias federales, estatales y municipales (DOF, 2013). Como parte de la $\mathrm{CNCH}$ se creó el Programa de Comedores Comunitarios (PCC), con el que se busca mejorar las condiciones nutricionales mediante la construcción y operación de comedores para la preparación y consumo de alimentos para la población seleccionada en el marco de la $\mathrm{CNCH}$ (Sedesol, 2015). Otro de los programas que coordinó la $\mathrm{CNCH}$ es el Programa de Albergues Escolares Indígenas (PAEI), el cual brinda alimentación a población de comunidades indígenas (CDI, 2019).

Como se verá enseguida, de este último subconjunto de programas que apoyan el consumo de alimentos, se seleccionaron cuatro (el PAL, PASL, Programa de Abasto Rural -PAR- y el Componente alimentario Prospera) para llevar a cabo un análisis estadístico de su intervención a nivel de las 32 entidades federativas entre 2010 y 2015, así como de su posible relación con el comportamiento de la carencia alimentaria en los estados. Esta selección obedeció a que, además de operar en los 32 estados del país, fueron considerados por el Coneval (2011a) como los principales programas orientados a atender problemas alimentarios y de abasto, y cuyo objetivo común es incidir en la mejora de la alimentación y 
nutrición, conformando el grupo denominado ayuda alimentaria y generación de capacidades. Antes de proceder al análisis estadístico, a continuación, se presentan algunas particularidades de cada uno de estos programas, derivadas de la revisión de estudios realizados en torno a su diseño, implementación y evaluación.

El PASL es un programa importante en las áreas urbanas, principalmente en la zona metropolitana de la Ciudad de México. Si bien cumple con el objetivo de apoyar a sus beneficiarios con leche fortificada a bajo costo, no hay indicadores que permitan medir su contribución a la nutrición de los beneficiarios (ASF, 2013). En cuanto al PAR, este ha incrementado sus objetivos y los servicios que brindan las Tiendas Diconsa. De acuerdo con la Auditoría Superior de la Federación (ASF, 2014), el programa ha cumplido con sus objetivos en términos de la adquisición de productos para abastecer las tiendas, generando un ahorro entre sus beneficiarios del $23.2 \%$. El programa cuenta con indicadores que permiten verificar el cumplimiento de sus objetivos (Coneval, 2011b).

Respecto a Progresa-Oportunidades-Prospera (POP), en la evaluación de su componente alimentario se concluyó que ha mejorado la alimentación de las personas que atiende y aunque su efectividad varía según el grupo poblacional, tiene importantes logros relacionados con la nutrición de niños, niñas y mujeres embarazadas (ASF, 2015). Por su parte, Bracamontes y Camberos (2019), quienes analizan el impacto de transferencias monetarias a través de remesas y del PDHO en Chiapas, Guerrero y Oaxaca durante el periodo 2010-2014, señalan que Oportunidades resultó más efectivo que las remesas: no obstante que su impacto sobre la pobreza alimentaria es sumamente limitado logró amortiguar la reducción del ingreso de sus beneficiarios en el marco de la crisis financiera global. Desde otro punto de vista, Tamez, Ruíz, Ayala y Ortíz (2019), quienes analizaron los efectos tanto de Oportunidades como del PAL, señalan que estos programas no han logrado impactar la calidad de vida de los beneficiarios debido a que tienen graves dificultades en cuanto a la identificación y selección de beneficiarios. En ese sentido Boltvinik, Damián, Jaramillo y de la Torre (2019) destacan la mala focalización del POP, pues calculan que más de la mitad de sus beneficiarios no padece pobreza extrema.

El PAL es un programa complementario de Prospera pues atiende a quienes pueden ser considerados como su población objetivo, pero no lo son, pues sus comunidades carecen de infraestructura educativa o de salud. Entrega apoyos 
económicos y tiene deficiencias en la medición del logro de sus objetivos, carece de mecanismos para dar seguimiento adecuado a sus beneficiarios y opera en comunidades donde no debiese hacerlo según sus reglas de operación (ROP) (ASF, 2012, 2015; Hospital Infantil de México Federico Gómez, 2009; INSP, 2015). Por el contrario, Huesca, López y Palacios (2016) han afirmado que su estrategia es exitosa ya que el PAL ha incrementado el bienestar de la población, no obstante, señalan que su cobertura y los recursos asignados son insuficientes para atender a las familias en situación de carencia alimentaria.

En conjunto, como se ha podido establecer, los programas trabajan sobre diversas poblaciones objetivo a través de diferentes estrategias para atender la falta de acceso a la alimentación de sus beneficiarios. Entre estas últimas se pueden distinguir dos vías de atención: apoyos económicos y provisión de productos de consumo básico a precios subsidiados. Si bien se considera que los programas son coherentes con el objetivo de promover el acceso a la alimentación, es necesaria una mayor coordinación para generar complementariedades y lograr un esfuerzo integral, así como retomar las fortalezas de los programas para reproducirlas e identificar sus debilidades para evitarlas (ASF, 2015, 2012; INSP, 2015; Hospital Infantil de México Federico Gómez, 2009). En general se encontró que los programas presentan deficiencias en términos de su diseño e implementación, lo cual disminuye su contribución al combate de la carencia de acceso a la alimentación. No obstante, pueden contribuir a reducir dicha carencia toda vez que se solventen las deficiencias señaladas en diversos estudios, tales como que Prospera no ha logrado modificar los hábitos alimenticios de sus beneficiarios, en particular de niños y niñas, por lo que estos siguen contrayendo enfermedades relacionadas a una mala nutrición (ASF, 2015); la reducción de la capacidad de autofinanciamiento del PASL dada la incorporación de beneficiarios que originalmente no estaban contemplados (ASF, 2012); la atención de población que no cumple con los criterios estipulados en las ROP del PAL (Hospital Infantil de México Federico Gómez, 2009), mientras desatiende a la mitad de su población objetivo, así como la necesidad de revaloración de los alimentos que entrega, los cuales debiesen ser elegidos acorde a las preferencias de los beneficiarios (INSP, 2015). 


\section{Intensidad de la intervención pública y cambios en la carencia alimentaria a nivel nacional y estatal, 2010-2015}

En el presente apartado se analizan los niveles de intervención de los programas sociales seleccionados en las entidades federativas, así como el comportamiento de la carencia alimentaria entre 2010 y 2015. Inicialmente, se presenta la metodología utilizada así como las fuentes de información mediante las cuales se obtuvieron indicadores en torno a la intensidad de la intervención de dichos programas. Posteriormente, se presentan los resultados del análisis.

\section{Metodología y fuentes de información}

Para llevar a cabo el análisis estadístico se conformaron tres indicadores: dos que miden la intensidad de la intervención de los programas a escala estatal entre 2010 y 2015 y uno que alude al comportamiento de la situación alimentaria en las entidades federativas durante el mismo periodo. Los dos primeros miden aspectos del grado promedio de la intervención de los programas entre 2010 y 2015: a) cobertura, medida a través del promedio registrado respecto de la población total de cada entidad federativa, y b) gasto, cuantificado mediante el promedio del gasto per cápita respecto a la población total estatal. El tercer indicador, el de carencia alimentaria, mide el cambio en la proporción de personas con carencia por acceso a la alimentación en cada estado entre 2010 y 2015.

Respecto a las fuentes de información, la proporción de carencia alimentaria fue obtenida del Coneval, organismo que mide el grado de seguridad alimentaria en las entidades federativas cada dos años, a partir de 2008 así como para $2015 .{ }^{3} \mathrm{La}$ proporción de carencia por acceso a la alimentación se calcula a partir de la Escala Mexicana de Seguridad Alimentaria (EMSA). ${ }^{4}$ De acuerdo con el Coneval (2015), un hogar puede tener un grado de inseguridad alimentaria severo, moderado o leve, o contar con seguridad alimentaria. Así, una persona carece de acceso a la alimentación si vive en un hogar que presenta inseguridad alimentaria moderada o severa. En cuanto a la información relativa a los programas, esta se obtuvo en su totalidad a través del Instituto Nacional de Transparencia, Acceso a la 
Información y Protección de Datos Personales (INAI), mediante solicitudes de información dirigidas a las instancias responsables de cada programa.

Se debe tener presente que cada uno de los indicadores fue calculado para cada entidad federativa y que, al hacer referencia a la variación relativa, se cuantifica el cambio de la variable descrita, en términos relativos, entre 2010 y 2015 , mientras que cuando se hace referencia a promedios, estos se habrán calculado para el mismo periodo. También es de mencionar que, para realizar comparaciones entre los montos de gasto, los cuales inicialmente estaban expresados en pesos corrientes, se procedió a convertirlos a precios constantes de 2010. Finalmente, cabe precisar que el análisis exploratorio consistió en la estimación de correlaciones, ${ }^{5}$ mediante las cuales se trató de identificar la relación existente entre la intensidad de la intervención de los programas sociales seleccionados y los niveles de carencia alimentaria de los estados. Como se verá más adelante, para este propósito se estimaron correlaciones entre los niveles de gasto y cobertura de cada programa, así como entre el gasto total y la cobertura del conjunto de programas. Posteriormente, con el fin de analizar la relación entre la carencia alimentaria de los estados y la intervención de los programas se estimaron correlaciones entre la variación absoluta de la carencia respecto a la variación absoluta del gasto del conjunto de los programas, así como de la variación absoluta de la cobertura del PAL, PASL y el Componente Alimentario Prospera. Para reforzar los resultados de tales correlaciones se procedió a estimar la relación entre la variación relativa de la carencia alimentaria y la variación relativa del gasto total promedio, así como con la variación relativa de la cobertura total de los programas.

\section{Intensidad de la intervención: el gasto per cápita y la cobertura de los programas}

Respecto a la intervención por parte de los programas en los estados, en el Cuadro 2 se muestran sus promedios de gasto y cobertura entre 2010 y 2015. Como puede apreciarse, en cuanto al gasto y la cobertura totales de los programas en estudio, ${ }^{6}$ para ambos indicadores las tres entidades que tuvieron los niveles de intervención más elevados fueron Chiapas, Oaxaca y Guerrero, es decir, los estados que concentran los mayores niveles de pobreza en el país. Es importante destacar que el comportamiento agregado de los cuatro programas a nivel estatal 
está fuertemente condicionado por la influencia del Componente alimentario Prospera, el cual aporta casi el $90 \%$ del gasto y el $87 \%$ de la cobertura para el conjunto de programas.

Cuadro 2.

Promedio anual del gasto per cápita y de la cobertura de los programas de apoyo al consumo de alimentos, por entidad federativa, 2010-2015

\begin{tabular}{|c|c|c|c|c|c|c|c|c|c|c|}
\hline \multirow[t]{2}{*}{$\begin{array}{c}\text { Entidad } \\
\text { federativa }\end{array}$} & \multicolumn{3}{|c|}{ PASL } & \multicolumn{3}{|c|}{$\begin{array}{l}\text { Componente } \\
\text { alimentario } \\
\text { PROSPERA }\end{array}$} & \multicolumn{2}{|c|}{ PAR } & \multicolumn{2}{|c|}{ Total } \\
\hline & $\begin{array}{c}\text { Gasto } \\
\left({ }^{*}\right)\end{array}$ & $\begin{array}{l}\text { Cobertur } \\
(* *)\end{array}$ & $\begin{array}{c}\text { Gasto } \\
\left({ }^{*}\right)\end{array}$ & $\begin{array}{c}\text { Cobertura } \\
\left({ }^{* *}\right)\end{array}$ & $\begin{array}{c}\text { Gasto } \\
(*)\end{array}$ & $\begin{array}{c}\text { Cobertur } \\
\left({ }^{* *}\right)\end{array}$ & $\begin{array}{l}\text { Gasto } \\
\left({ }^{*}\right)\end{array}$ & $\begin{array}{c}\text { Cobertura } \\
(* *)\end{array}$ & $\begin{array}{l}\text { Gasto } \\
\left({ }^{*}\right)(* *)\end{array}$ & $\begin{array}{c}\text { Cobertura } \\
\left({ }^{* *}\right)\left({ }^{* * *}\right)\end{array}$ \\
\hline \multicolumn{11}{|c|}{ Estado con nivel de gasto muy alto } \\
\hline Chiapas & $2.87^{\circ}$ & 2.85 & 79.88 & 5.65 & 680.99 & 57.78 & 24.88 & 0.04 & 763.73 & 66.28 \\
\hline Oaxaca & 6.62 & 3.41 & 67.91 & 3.96 & 646.34 & 45.02 & 37.72 & 0.06 & 720.87 & 52.39 \\
\hline Guerrero & 7.59 & 4.6 & 45.11 & 3.12 & 641.31 & 52.13 & 19.97 & 0.04 & 694.01 & 59.84 \\
\hline \multicolumn{11}{|c|}{ Estado con nivel de gasto alto } \\
\hline Puebla & 3.77 & 2.63 & 28.44 & 2.19 & 434.34 & 34.46 & 12.27 & 0.02 & 466.55 & 39.28 \\
\hline Veracruz & 4.37 & 2.75 & 30.73 & 1.88 & 423.47 & 34.09 & 20.29 & 0.03 & 458.56 & 38.73 \\
\hline Tabasco & 4.67 & 2.61 & 34.62 & 1.99 & 418.17 & 34.72 & 36.37 & 0.04 & 457.46 & 39.32 \\
\hline Campeche & 5.28 & 3.70 & 45.07 & 2.79 & 404.18 & 29.55 & 37.58 & 0.05 & 454.52 & 36.04 \\
\hline Hidalgo & 11.97 & 7.24 & 22.82 & 1.85 & 415.34 & 32.57 & 26.26 & 0.03 & 450.13 & 41.66 \\
\hline Michoacán & 7.97 & 4.51 & 45.73 & 3.37 & 360.84 & 28.47 & 8.72 & 0.02 & 414.54 & 36.35 \\
\hline $\begin{array}{l}\text { San Luis } \\
\text { Potosí }\end{array}$ & 8.06 & 4.63 & 6.78 & 0.61 & 399.04 & 32.54 & 21.19 & 0.04 & 413.88 & 37.77 \\
\hline \multicolumn{11}{|c|}{ Estado con nivel de gasto medio } \\
\hline Yucatán & 5.98 & 2.83 & 10.52 & 0.74 & 373.79 & 29.93 & 37.52 & 0.03 & 390.29 & 33.50 \\
\hline Zacatecas & 13.31 & 7.21 & 14.26 & 0.99 & 341.23 & 29.01 & 82.95 & 0.04 & 368.80 & 37.22 \\
\hline Tlaxcala & 10.41 & 5.71 & 12.80 & 1.02 & 330.59 & 27.16 & 9.94 & 0.02 & 353.79 & 33.89 \\
\hline Durango & 9.28 & 5.71 & 26.19 & 2.25 & 308.28 & 25.07 & 31.39 & 0.05 & 343.75 & 33.02 \\
\hline $\begin{array}{l}\text { Quintana } \\
\text { Roo }\end{array}$ & 4.75 & 2.82 & 48.86 & 3.10 & 263.09 & 18.13 & 23.43 & 0.03 & 316.70 & 24.06 \\
\hline \multicolumn{11}{|c|}{ Estado con nivel de gasto bajo } \\
\hline Querétaro & 6.69 & 3.96 & 44.09 & 3.64 & 243.49 & 18.00 & 13.90 & 0.02 & 294.27 & 25.60 \\
\hline Sinaloa & 3.09 & 2.66 & 18.69 & 1.03 & 266.57 & 21.25 & 19.49 & 0.03 & 288.35 & 24.95 \\
\hline Guanajuato & 15.54 & 3.80 & 28.15 & 2.36 & 242.28 & 21.14 & 6.32 & 0.02 & 285.97 & 27.29 \\
\hline Morelos & 10.60 & 6.48 & 18.53 & 1.01 & 252.21 & 19.17 & 10.30 & 0.01 & 281.34 & 26.66 \\
\hline México & 17.83 & 11.96 & 51.56 & 4.39 & 201.17 & 14.46 & 3.68 & 0.01 & 270.56 & 30.81 \\
\hline Chihuahua & 2.88 & 2.17 & 67.11 & 4.01 & 178.29 & 12.16 & 19.00 & 0.02 & 248.28 & 18.34 \\
\hline Nayarit & 17.45 & 9.02 & 15.41 & 1.24 & 209.66 & 16.43 & 45.17 & 0.05 & 242.52 & 26.69 \\
\hline Tamaulipas & 1.57 & 1.28 & 33.35 & 2.23 & 206.14 & 15.34 & 11.14 & 0.02 & 241.05 & 18.86 \\
\hline
\end{tabular}




\section{EVOLUCIÓN DE LA POLÍTICA DE APOYO A LA ALIMENTACIÓN EN LAS ALTERNANCIAS POLÍTICAS \\ Y EL CAMBIO EN LA SITUACIÓN ALIMENTARIA \\ EN LAS ENTIDADES FEDERATIVAS MEXICANAS ENTRE 2010 Y 2015}

BECERRA-MONTES, ORDÓŃEZ-BARBA

\begin{tabular}{|c|c|c|c|c|c|c|c|c|c|c|}
\hline $\begin{array}{l}\text { Baja } \\
\text { California Sur }\end{array}$ & 4.13 & 3.83 & 46.97 & 3.72 & 174.30 & 11.81 & 26.37 & 0.03 & 225.40 & 19.37 \\
\hline Sonora & 2.46 & 2.29 & 29.71 & 2.18 & 181.29 & 13.05 & 12.80 & 0.02 & 213.46 & 17.53 \\
\hline \multicolumn{11}{|c|}{ Estado con nivel de gasto muy bajo } \\
\hline Colima & 15.02 & 4.20 & 10.72 & 1.04 & 168.63 & 12.93 & 15.48 & 0.01 & 194.37 & 18.18 \\
\hline Jalisco & 6.76 & 4.16 & 27.26 & 2.87 & 136.36 & 9.99 & 5.34 & 0.01 & 170.38 & 17.03 \\
\hline $\begin{array}{l}\text { Ciudad de } \\
\text { México }\end{array}$ & 23.09 & 11.49 & 62.91 & 3.80 & 72.20 & 1.27 & 24.35 & 0.00 & 158.20 & 16.55 \\
\hline Aguascalientes & 6.46 & 3.94 & 9.14 & 0.69 & 130.69 & 12.17 & 1.23 & 0.00 & 146.28 & 16.80 \\
\hline Coahuila & 4.42 & 2.35 & 13.29 & 1.05 & 115.11 & 8.72 & 13.31 & 0.02 & 132.82 & 12.12 \\
\hline $\begin{array}{l}\text { Baja } \\
\text { California }\end{array}$ & 1.96 & 1.38 & 36.09 & 3.19 & 91.96 & 5.89 & 6.65 & 0.00 & 130.00 & 10.46 \\
\hline Nuevo León & 1.33 & 1.24 & 10.48 & 0.93 & 76.55 & 6.18 & 9.71 & 0.01 & 88.36 & 8.36 \\
\hline Promedio & 7.8 & 4.4 & 32.6 & 2.3 & 293.4 & 22.8 & 21.1 & 0.0 & 333.7 & 29.5 \\
\hline
\end{tabular}

Fuente: elaboración propia con datos de Coneval, INEGI, Coordinación Nacional de Prospera, Liconsa y Diconsa (2010-2015), a precios de 2010. Nota: los estados están ordenados de mayor a menor gasto per cápita promedio total registrado al 2015. *Promedio entre 2010 y 2015, expresado en pesos per cápita. ${ }^{* *}$ Promedio entre 2010 y 2015, expresado en porcentaje respecto a la población de la entidad. ${ }^{* * *}$ No se contabiliza la cobertura y gasto del PAR debido a diferencias en el cálculo de su cobertura respecto de los otros programas.

De esta forma, los tres programas restantes muestran tendencias diferentes: en el caso del PASL, su cobertura y gasto fueron mayores en Ciudad de México, Estado de México y Nayarit; en el PAL los estados con mayor gasto per cápita promedio fueron Chiapas, Oaxaca y Chihuahua, mientras que en la cobertura los mayores niveles estuvieron en Chiapas, Chihuahua y México; finalmente, para el PAR se aprecia que Zacatecas, Oaxaca y Campeche registraron los mayores gastos promedio entre 2010 y 2015, mientras que en cobertura fueron mayormente atendidos Oaxaca, Nayarit y Durango.

Cabe mencionar que respecto al gasto total per cápita de los cuatro programas, entre 2010 y 2015 se presentó un incremento insignificante de $0.31 \%$, al pasar de $\$ 11,731.21$ a $\$ 11,767.12$; no obstante, tanto el PASL como el Componente alimentario de Prospera tuvieron una reducción de su gasto per cápita del -20.9 y 3.89 \%, respectivamente. Por el contrario, tanto el PAL (34.56 \%) como el PAR $(19.05 \%)$ tuvieron incrementos importantes en su gasto per cápita. Por otro lado, respecto a la cobertura total del conjunto de los cuatro programas, esta presentó una reducción en términos relativos $(-7.67 \%)$ entre 2010 y 2015, al pasar de 
31.68 a $29.25 \%$. Debe señalarse que entre los programas hubo un comportamiento diferenciado toda vez que tanto el PAL (-27.47\%) como el Componente alimentario Prospera $(-6.74 \%)$ mostraron una reducción en su cobertura, mientras que el PAR $(8.93 \%)$ y el PASL $(0.38 \%)$ tuvieron una expansión entre 2010-2015.

Respecto al análisis correlacional, inicialmente se estimó la correlación entre la cobertura y el gasto per cápita promedio de los programas, cuyos resultados se observan en la Figura 1. En cuanto al PASL su coeficiente de correlación fue de 0.894, es decir, la cobertura del programa en los estados responde positivamente al incremento del gasto asignado. La misma relación (positiva) se puede apreciar en el caso del PAL, Componente alimentario Prospera y del PAR, pues estos presentaron un coeficiente de $0.954,0.990$ y 0.661 , respectivamente.
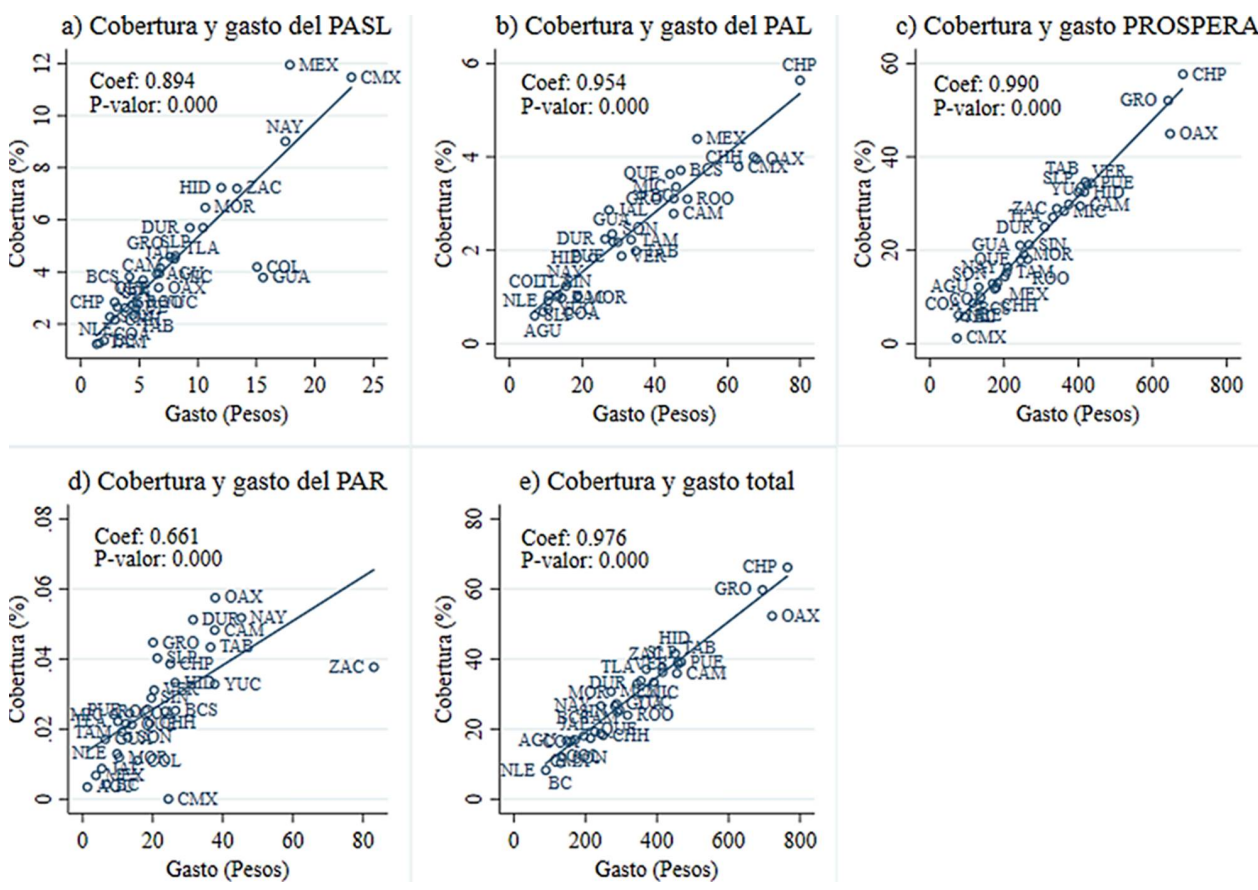

Figura 1. Diagramas de dispersión de los promedios de la cobertura y el gasto per cápita de los programas de apoyo a la alimentación para las entidades federativas de México, 2010-2015.

Fuente: elaboración propia con datos de Coneval, INEGI, Coordinación Nacional de Prospera, Liconsa y Diconsa (2010-2015), a precios de 2010 Nota: Las cifras correspondientes a la cobertura y gasto totales se calcularon como la suma de los programas sociales con excepción del PAR. 
En el caso del PASL se puede ver que tanto Ciudad de México, como el Estado de México y Nayarit son las entidades donde tuvo una mayor expansión de su cobertura dado su gasto asignado. Respecto al PAL, Chiapas, México y Oaxaca son los estados que durante el periodo de análisis incrementaron su cobertura en mayor medida que el resto de los estados. En cuanto al Componente alimentario de Prospera, Chiapas, Guerrero y Oaxaca fueron los que expandieron mayormente su cobertura derivado del gasto que les fue asignado; finalmente, en el caso del PAR, los estados cuya cobertura aumentó en mayor grado fueron Zacatecas, Oaxaca, Nayarit, Durango y Campeche.

Por su parte, en la correlación entre la cobertura y el gasto total se aprecia en la Figura 1 inciso e, una relación positiva con un coeficiente de 0.976, es decir, que la cobertura incrementó dado el incremento del gasto; por estados, Chiapas, Guerrero y Oaxaca mostraron un mayor incremento en la cobertura por parte de los programas considerando el incremento en el gasto conjunto del PAL, PASL y del Componente alimentario Prospera.

Cambio en la proporción de la carencia alimentaria a nivel nacional y estatal

Como puede apreciarse en el Cuadro 3, las entidades federativas en conjunto presentaron una variación relativa promedio de $-8.9 \%$ entre 2010 y 2015, es decir, que hubo una reducción en la proporción de carencia alimentaria en el país al pasar el promedio a nivel nacional de 24.85 a $21.74 \%$ en dicho periodo. No obstante, dada la heterogeneidad entre los estados, hubo algunos que mostraron un notable aumento como Tamaulipas (44.1\%), Oaxaca (23.1\%) y Tabasco (19.4 \%), mientras que San Luis Potosí (-44 \%), Ciudad de México (-36 \%) y el Estado de México (-36 \%) presentaron la mayor reducción en su proporción de carencia. En total hubo 24 entidades que redujeron sus niveles de carencia, mientras que ocho los aumentaron. 


\section{ESTUDIOS SOCIALES}

Cuadro 3.

Posición y proporción de carencia alimentaria entre 2010 y 2015

\begin{tabular}{|c|c|c|c|c|c|c|}
\hline \multirow{2}{*}{ Entidad federativa } & \multicolumn{2}{|c|}{2010} & \multicolumn{2}{|c|}{2015} & \multicolumn{2}{|c|}{ Variación } \\
\hline & Posición & Carencia (\%) & Posición & Carencia (\%) & Absoluta & Relativa \\
\hline Guerrero & 1 & 42.7 & 1 & 40.6 & -2.0 & -4.7 \\
\hline Tabasco & 2 & 33.3 & 2 & 39.8 & 6.5 & 19.4 \\
\hline Oaxaca & 10 & 26.4 & 3 & 32.5 & 6.1 & 23.1 \\
\hline Michoacán & 8 & 28.8 & 4 & 32.0 & 3.2 & 11.1 \\
\hline Veracruz & 11 & 26.1 & 5 & 30.1 & 4.0 & 15.2 \\
\hline Morelos & 20 & 22.0 & 6 & 26.2 & 4.2 & 19.3 \\
\hline Guanajuato & 17 & 23.7 & 7 & 26.1 & 2.4 & 10.1 \\
\hline Chiapas & 5 & 30.3 & 8 & 25.0 & -5.3 & -17.4 \\
\hline Puebla & 9 & 27.6 & 9 & 24.0 & -3.6 & -13.1 \\
\hline Sonora & 13 & 25.8 & 10 & 23.7 & -2.1 & -8.2 \\
\hline Sinaloa & 15 & 24.5 & 11 & 23.3 & -1.2 & -4.9 \\
\hline Campeche & 4 & 31.2 & 12 & 23.2 & -8.0 & -25.7 \\
\hline Quintana Roo & 21 & 21.8 & 13 & 22.2 & 0.4 & 2.0 \\
\hline Baja California Sur & 12 & 26.0 & 14 & 21.7 & -4.3 & -16.6 \\
\hline Hidalgo & 7 & 29.0 & 15 & 21.6 & -7.4 & -25.6 \\
\hline Tlaxcala & 16 & 24.2 & 16 & 21.5 & -2.6 & -10.9 \\
\hline México & 3 & 31.6 & 17 & 20.2 & -11.4 & -36.0 \\
\hline Zacatecas & 14 & 24.9 & 18 & 20.0 & -4.9 & -19.5 \\
\hline Tamaulipas & 32 & 13.6 & 19 & 19.6 & 6.0 & 44.1 \\
\hline Colima & 27 & 19.9 & 20 & 19.5 & -0.3 & -1.7 \\
\hline Nayarit & 18 & 23.6 & 21 & 19.5 & -4.1 & -17.5 \\
\hline Aguascalientes & 26 & 20.2 & 22 & 18.8 & -1.4 & -7.0 \\
\hline Durango & 25 & 20.3 & 23 & 17.8 & -2.4 & -12.1 \\
\hline Queretaro & 23 & 21.3 & 24 & 17.5 & -3.8 & -17.9 \\
\hline Yucatán & 22 & 21.4 & 25 & 16.9 & -4.5 & -21.1 \\
\hline San Luis Potosí & 6 & 30.1 & 26 & 16.9 & -13.2 & -44.0 \\
\hline Coahuila & 24 & 20.8 & 27 & 16.3 & -4.4 & -21.4 \\
\hline Nuevo León & 30 & 15.7 & 28 & 15.5 & -0.1 & -0.7 \\
\hline Jalisco & 19 & 22.0 & 29 & 15.2 & -6.8 & -30.8 \\
\hline Baja California & 29 & 16.4 & 30 & 14.9 & -1.4 & -8.6 \\
\hline Chihuahua & 28 & 17.7 & 31 & 12.9 & -4.8 & -26.9 \\
\hline Ciudad de México & 31 & 15.5 & 32 & 9.9 & -5.6 & -36.1 \\
\hline Promedio & & 24.8 & & 21.7 & -2.3 & -8.9 \\
\hline
\end{tabular}

Fuente: elaboración propia con datos de Coneval (2015). Nota: Entidades federativas ordenadas en orden descendente de acuerdo con la proporción de carencia alimentaria estatal al 2015. 
Tanto en 2010 como en 2015 los estados con mayores proporciones de carencia alimentaria fueron Guerrero y Tabasco, pero sorprende que estados como Oaxaca, Michoacán, Veracruz, Morelos y Guanajuato hayan modificado su posición negativamente en 2015, empeorando significativamente con ello el problema alimentario que ya padecían en 2010. En varios de estos casos, como veremos enseguida, el deterioro se produjo a pesar de haber sido beneficiarios privilegiados de los programas alimentarios.

\section{Carencia alimentaria e intervención de los programas de acceso a la alimentación}

En la Figura 2, incisos a y b, se aprecia la relación entre la variación absoluta de la carencia respecto de la variación absoluta del gasto total promedio y de la cobertura total de tres programas de apoyo al consumo de alimentos, es decir, el PAL, el PASL y el Componente Alimentario Prospera. ${ }^{7}$ Los resultados indican, según el inciso a, que el coeficiente de correlación respecto al gasto total es de $0.1230 \mathrm{y}$, aunque la relación es positiva, ésta no es significativa pues su p-valor es de 0.5024 . Por su parte, en el inciso b se puede apreciar que la relación respecto a la variación de la cobertura es igualmente positiva $(0.1985)$ y no significativa dado el p-valor de 0.2761. En otras palabras, en ninguno de los dos casos los cambios en la intervención de los programas tienen efectos sobre la disminución o el aumento de la carencia alimentaria a nivel estatal. 

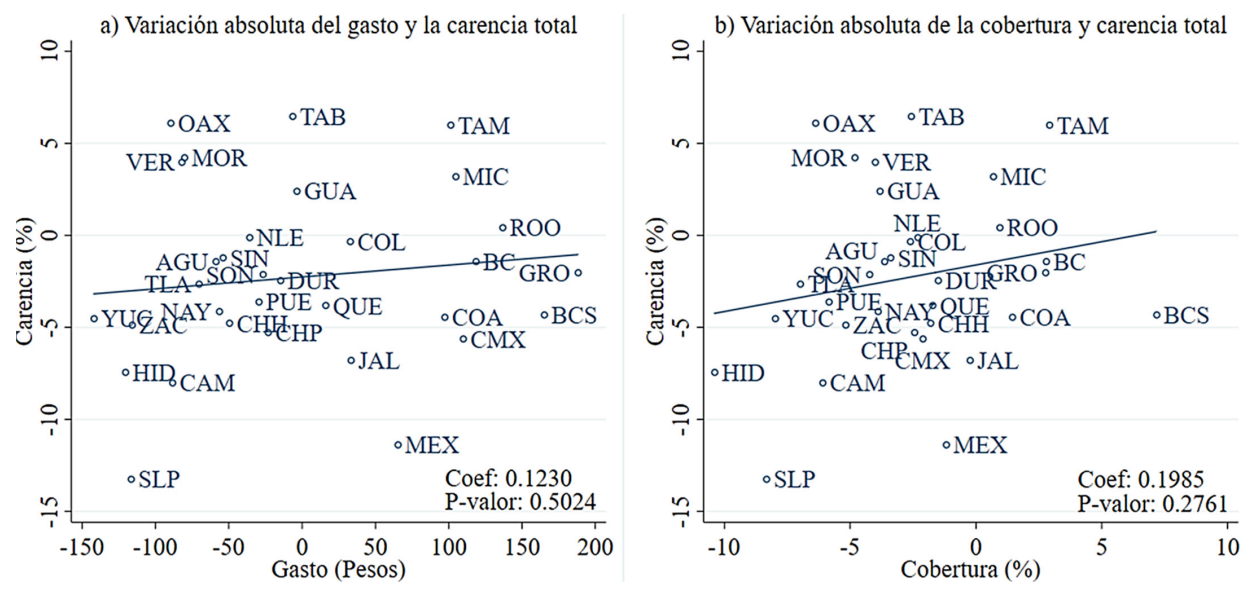

Figura 2. Diagrama de dispersión de la variación absoluta de la carencia frente a la variación absoluta del gasto (a precios de 2010) y de la cobertura total de los programas PAL, PASL y Componente Alimentario PROSPERA

Fuente: elaboración propia con datos de Coneval, INEGI, Coordinación Nacional de Prospera y Liconsa (2010-2015), a precios de 2010.

Para confirmar los resultados anteriores, se procedió a valorar la relación entre la variación relativa de la carencia alimentaria respecto de la variación relativa del gasto total promedio, así como de la variación relativa de la cobertura total de los programas. En el primer caso se puede apreciar en la Figura 3 inciso a, que el coeficiente estimado fue de 0.1013 , aunque este no es significativo dado su p-valor (0.5813). Igualmente, se aprecia en la gráfica b que la relación entre la variación relativa de la carencia y la cobertura total de los programas fue positiva (0.1351), pero tampoco es significativa. 

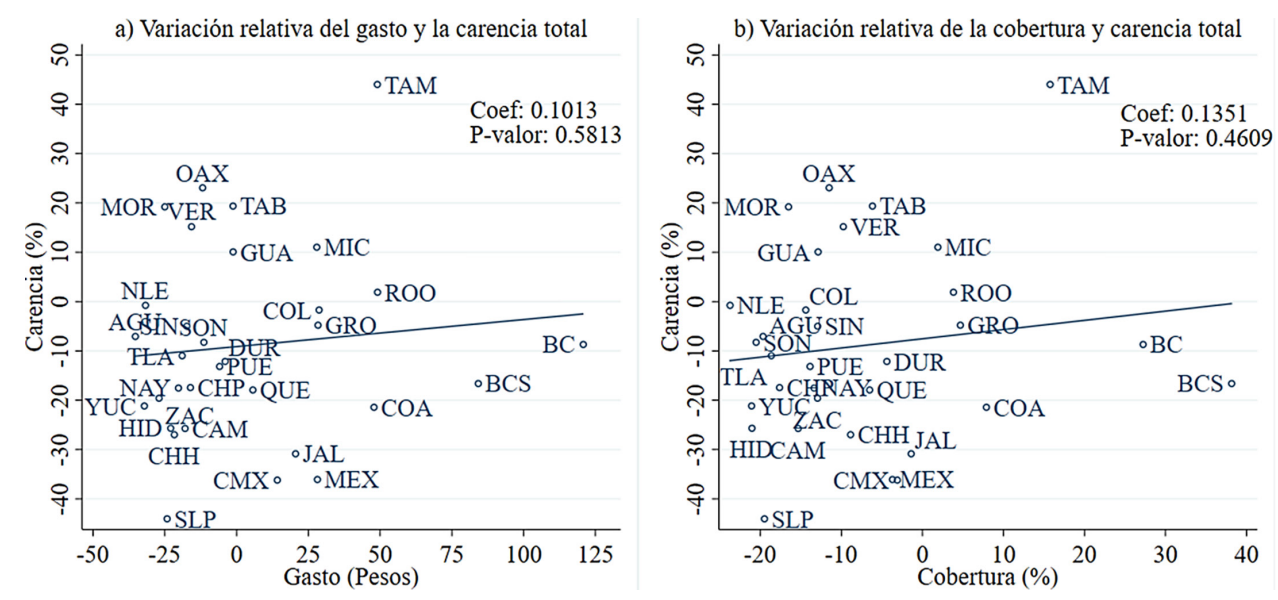

Figura 3. Diagrama de dispersión de la variación relativa de la carencia frente a la variación relativa del gasto (a precios de 2010) y la cobertura total de los programas PAL, PASL y Componente Alimentario PROSPERA

Fuente: elaboración propia con datos de Coneval, INEGI, Coordinación Nacional de Prospera y Liconsa (2010-2015), a precios de 2010.

Con los resultados anteriores se puede concluir que la intervención de los tres programas no logró incidir sobre el cambio en la carencia alimentaria en las entidades federativas entre 2010 y 2015. Como puede apreciarse en los Cuadros 2 y 3, a nivel de cada estado hay entidades que tuvieron los mayores niveles de cobertura y gasto promedio, y contrariamente a lo esperado tuvieron fuertes aumentos en sus niveles de carencia, como en los casos de Tabasco, Oaxaca y Veracruz. Entre este grupo debe resaltarse que en el caso de Oaxaca el incremento de la proporción de carencia en el periodo fue por demás alarmante, toda vez que pasó de ubicarse en la posición 10 en 2010 a ocupar el lugar 3 en 2015, esto aún a pesar que la cobertura de los tres programas fue superior al $52 \%$ de la población oaxaqueńa. Por el contrario, hubo entidades que recibieron cantidades muy inferiores de presupuesto y, por ende, tuvieron una cobertura por debajo del $18 \%$ y lograron reducir significativamente la carencia alimentaria en el periodo en estudio; en la situación más extrema de esta tendencia se encuentran Coahuila, Jalisco y Ciudad de México, que tuvieron descensos relativos de entre 21 y $36 \%$ en sus niveles de carencia entre 2010 y 2015. 


\section{Conclusiones}

La política alimentaria ha cambiado poco a lo largo de las tres administraciones más recientes. Aunque cada una introdujo ciertas particularidades, en términos generales, la política se mantuvo sobre las líneas trazadas en las dos últimas décadas del siglo XX. Ello es, se centraron en la aplicación de programas con objetivos alimentarios altamente focalizados y haciendo uso de nuevas modalidades de apoyos como las Transferencias Monetarias Condicionadas (TMC), de los apoyos dirigidos hacia grupos vulnerables (mujeres embarazadas, nińos en edad escolar, adultos mayores, etc.) y de los subsidios orientados a un grupo reducido de alimentos. En este periodo, el programa central de la política alimentaria mexicana fue hasta 2018 el POP, pero recientemente fue sustituido por el gobierno de López Obrador por un programa de becas a estudiantes de preparatorias públicas.

En este contexto, el análisis sobre la eficacia de los programas de apoyo a la alimentación en cuanto a su capacidad para reducir la carencia alimentaria en los estados indica que, a pesar de haberse observado un caída en la carencia a nivel nacional (de $-12.4 \%$ en términos relativos) y en la mayoría de las entidades federativas (24 en total) entre 2010 y 2015, ésta no tuvo mucho que ver con la intensidad de la intervención en cada estado. Así, se encontró que lugares como Tabasco, Oaxaca o Veracruz, que estuvieron entre las seis entidades más atendidas por los programas alimentarios, tuvieron aumentos importantes en su carencia alimentaria. Por el contrario, algunos de los estados menos apoyados, como Coahuila, Jalisco y Ciudad de México, tuvieron descensos significativos en sus niveles de carencia entre 2010 y 2015.

Una parte de estos resultados puede deberse a deficiencias en la operación de los programas o a la manera en cómo distribuyen sus recursos a escala territorial. Derivado del análisis del gasto y la cobertura de los programas se obtuvo que el PASL registró al 2015 sus más altos niveles de gasto per cápita y de cobertura en Ciudad de México, a pesar de que ese año fue el estado con menor proporción de carencia. Por otro lado, el gasto per cápita del PAL tuvo el incremento más considerable de entre los cuatro programas, no obstante haber mostrado deficiencias en cuanto a la medición del logro de sus objetivos, de operar en comunidades donde no debiese hacerlo y carecer de mecanismos para dar seguimiento a sus beneficiarios (ASF, 2012, 2015; Hospital Infantil de México 
Federico Gómez, 2009; INSP, 2015). Hacia 2015 su gasto se enfocó en estados con nivel de carencia muy alto y medio.

En cuanto al Componente alimentario de Prospera, su gasto per cápita y cobertura fueron en 2015 considerablemente mayores en Chiapas, Guerrero y Oaxaca, estados que tenían en ese año altos niveles de carencia alimentaria. El PAR también experimentó un incremento en su gasto total y asignó en 2015 un gasto muy superior a Zacatecas, Colima y Baja California Sur, estados con niveles medios de carencia. Respecto a su cobertura, Oaxaca, Nayarit y Durango fueron los que tuvieron mayor proporción; mostraba, el primero, un nivel de carencia alimentaria alto y los dos últimos niveles medios.

Derivado de lo anterior, se puede concluir que los programas analizados no comparten un patrón de distribución de recursos específico, pues hay programas (Componente alimentario Prospera) que parecen dirigir sus recursos, principalmente, hacia estados con altas proporciones de carencia alimentaria, mientras que otros optan por orientarlos a entidades federativas con bajos niveles de carencia, como el caso del PASL que tanto en 2010 como en 2015 dirigió sus recursos, particularmente, a Ciudad de México. Cabe resaltar, además, que el Componente alimentario Prospera no ha demostrado tener un efecto importante en la reducción de la carencia alimentaria en las entidades federativas, además de que los estados a los que destinó mayor presupuesto mantuvieron la misma posición en cuanto a su proporción de carencia (Guerrero y Tabasco) o incluso retrocedieron, como en el emblemático estado de Oaxaca.

Los resultados obtenidos en este análisis sugieren una revisión de los criterios bajo los cuales se orienta la operación del PASL, dado que como se pudo constatar, este programa concentró en 2010 y 2015 sus recursos en estados con proporción de carencia alimentaria muy inferior al promedio, pero principalmente a Ciudad de México. En ese sentido, es necesario recordar que, de acuerdo con Ávila et al. (2011), el PASL es un programa importante para las zonas urbanas donde se concentran principalmente sus beneficiarios, por lo que el Centro de Estudios Estratégico (2016) ha indicado que es prioritario que atienda igualmente a la población rural. Al igual que el PASL, la estimación de las correlaciones de la intervención del PAL, respecto a la carencia no mostraron tener relación alguna entre su gasto ni su cobertura. Cabe recordar que algunos autores (ASF, 2012, 2015; Hospital Infantil de México Federico Gómez, 2009; INSP, 2015) han 
mencionado que este programa carece de mecanismos para dar seguimiento a los beneficiarios y no opera con estricto apego a sus Reglas de Operación, y según Huesca, López y Palacios (2016), tanto su cobertura como sus recursos son insuficientes, lo cual se podría considerar una posible explicación a su ineficacia para atender la carencia alimentaria.

Debemos destacar que todos los programas mostraron un incremento en su cobertura y gasto, pero aun así el resultado principal del análisis es que la intervención federal a través de los programas analizados no tuvo un efecto significativo sobre la disminución de la proporción de la carencia alimentaria de las entidades federativas entre 2010 y 2015. Cabe subrayar que, aunque estos resultados se obtienen de un análisis exploratorio, pueden servir de base para próximas investigaciones en las que se busque determinar qué otros factores del entorno económico o social limitan la capacidad de intervención de los programas de apoyo al consumo de alimentos. En ese sentido, de acuerdo con Cuéllar (2011) para combatir la falta de acceso a la alimentación es importante tener presente las variables macroeconómicas, tales como la inflación, el tipo de cambio y las tasas de interés, que intervienen en las decisiones de consumidores y productores de alimentos y que los resultados que obtienen los programas para promover el acceso a alimentos se ven determinados por dichas variables. Además, según la FAO (2014), muchos de los esfuerzos contra los problemas alimentarios son intervenciones de corto plazo que buscan dar atención inmediata al problema de la falta de acceso a alimentos sin atender las cuestiones estructurales que causan dicho problema, lo cual disminuye las posibilidades de éxito de dichas intervenciones. Desde este punto de vista, siguiendo con el argumento de Cuéllar (2011), es necesario que se superen los problemas estructurales a través de la promoción de políticas nacionales e internacionales concatenadas que busquen atender el problema de la falta de alimentación con acciones a corto y largo plazo. Finalmente, se considera que ante los cambios que está promoviendo el gobierno federal actual resulta necesario mantener una postura crítica sobre el rumbo que tome la política alimentaria, pues si bien las acciones tomadas constituyen un hipotético esfuerzo para mejorar la implementación de los programas sociales, el optar por prácticas del pasado y aplicarlas en contextos diferentes representa un riesgo; no obstante, también puede ser una buena oportunidad para asegurar que 
los mexicanos que hasta ahora han visto limitado su acceso a la alimentación puedan finalmente ejercer este derecho.

Notas al pie:

1 Dicho programa sería renombrado en 2003 como Programa de Desarrollo Humano Oportunidades (PDHO) y en 2015 como Programa de Inclusión Social Prospera (Prospera). A partir del 28 de febrero de 2019 fue reemplazado por el Programa Beca Universal para Estudiantes de Educación Media Superior Benito Juárez.

${ }^{2}$ Organismo resultado de la fusión de Diconsa y Liconsa, empresas encargadas de la implementación del PAR y del PASL, respectivamente.

${ }^{3}$ El grado de inseguridad alimentaria se determina de acuerdo al número de preguntas contestadas de forma afirmativa durante la aplicación del Módulo de Condiciones Socioeconómicas de la Encuesta Nacional de Ingreso Gasto (MCS-ENIGH), a cargo del Instituto Nacional de Estadística y Geografía (INEGI).

${ }^{4}$ Se considera si durante los últimos tres meses, por falta de dinero u otros recursos los hogares tuvieron una alimentación con muy poca variedad de alimentos; dejaron de desayunar, comer o cenar; comieron menos de lo que consideran deberían de comer; alguna vez se quedaron sin comida; sintieron hambre, pero no comieron o comieron una vez al día o dejaron de comer todo un día. En hogares donde viven menores de edad, se realizan seis preguntas adicionales (Coneval, 2015).

${ }^{5}$ Mediante el coeficiente de correlación es posible medir la dirección y la fuerza o grado de asociación lineal existente entre dos variables, aunque no se establece la existencia de una relación entre ambas. El coeficiente puede tomar cualquier valor entre -1 y 1 , donde un valor igual a 1 indica una correlación lineal positiva perfecta.

${ }^{6}$ No se incluye la contribución del PAR en la medición del gasto y cobertura totales, dado que atiende a población abierta de las comunidades donde opera, por lo que carece de un padrón de beneficiarios y por ende el cálculo de su cobertura difiere del resto de programas. El PAR cuenta con un registro del número total de sus tiendas, a partir del cual es posible calcular su cobertura.

${ }^{7}$ El PAR no fue contabilizado en la construcción de las variables con las que se estimaron estas correlaciones debido a que el cálculo de su cobertura es diferente al del resto de los programas 


\section{Bibliografía}

Auditoría Superior de la Federación (ASF) (2015). Evaluación de la política pública de Prospera programa de inclusión social. Recuperado de: https://www.asf.gob.mx/ Trans/Informes/IR2015i/Documentos/Auditorias/2015_1575_a.pdf

ASF (2013). Liconsa, S. A. de C. V. Programa de Abasto Social de Leche Auditoría de Desempeño: 13-2-20VST-07-0268 DS-089. Recuperado de: https://www.asf.gob.mx/ Trans/Informes/IR2013i/Documentos/Auditorias/2013_0268_a.pdf

ASF (2012). Evaluación número 414 Apoyo alimentario. Recuperado de: https:/www.asf.gob.mx/Trans/Informes/IR2012i/Documentos/Auditorias/2012_041 4_a.pdf

Ávila, A., Flores, J. y Rangel, G. (2011). La politica alimentaria en México. México, D. F.: Centro de Estudios para el Desarrollo Rural Sustentable y la Soberanía Alimentaria.

Barba, C. (2016). Las propuestas de reformas y las nuevas estrategias sociales del gobierno de Peña Nieto ¿Cambio de rumbo en la política social mexicana? En: E. Valencia y G. Ordóñez (Eds.), Nueva ronda de reformas estructurales en México ¿Nuevas politicas sociales? Tijuana: El Colegio de la Frontera.

Barquera, S., Rivera-Dommarco, J. y Gasca-García A. (2001). Políticas y programas de alimentación y nutrición en México, Salud Pública, (43), 464-477. Recuperado de: http://www.scielo.org.mx/scielo.php?script=sci_arttext\&pid=S003636342001000500011

Boltvinik, J. (2016). Conceptos y mediciones de pobreza y políticas públicas. La medición multidimensional del Coneval y la Cruzada Nacional Contra el Hambre. En: E. Valencia y G. Ordóñez (Eds.), Nueva ronda de reformas estructurales en México, ¿nuevas políticas sociales? (pp. 422-461). Tijuana, México: El Colegio de la Frontera Norte.

Boltvinik, J., Damián, A.,Jaramillo, M. y de la Torre, R. (2019). Crónica de un fracaso anunciado. Ha llegado la hora de remplazar el Progresa-Oportunidades-Prospera (POP), en L. Huesca, G. Ordóńez y S. Sandoval (coordinadores), Los retos de la política social en el sexenio de la Cuarta Transformación. Tijuana, México: El Colegio de la Frontera Norte /Centro de Investigación en Alimentación y Desarrollo (en prensa). 
Bracamontes, J. y Camberos, M. (2019). El impacto de las remesas y el Programa Oportunidades después de la crisis global en la pobreza alimentaria de hogares urbano/rurales en Chiapas, Guerrero y Oaxaca, 2010-2014. En: L. Huesca, G. Ordóńez y S. Sandoval (coordinadores), Los retos de la política social en el sexenio de la Cuarta Transformación. Tijuana, México: El Colegio de la Frontera Norte /Centro de Investigación en Alimentación y Desarrollo (en prensa).

Brachet-Márquez, V. y Sherraden, M. (1993). Austeridad fiscal, el Estado de Bienestar y el cambio político: los casos de la salud y la alimentación en México (1970-1990). Estudios Sociológicos, Colmex (32).

Cardozo, M. (2005). Neoliberalismo y eficiencia de los programas sociales en México. Política y Cultura (24), 169-186. Recuperado de: http://biblat.unam.mx/en/revista/ politica-y-cultura/articulo/neoliberalismo-y-eficiencia-de-los-programas-sociales-enmexico

Centro de Estudios Estratégicos (CEE), (2006). Diseño del Programa de Abasto Social de Leche.

Comisión Nacional para el Desarrollo de los Pueblos Indígenas (CDI) (2019). Programa Albergues Escolares Indigenas. Recuperado de: http://www.cdi.gob.mx/focalizada/paei/ index.php

Consejo Nacional de Evaluación de la Política de Desarrollo Social (Coneval) (2018). Medición de la pobreza. Resultados de pobreza en México 2018 a nivel nacional y por entidades federativas. Recuperado de: https://www.coneval.org.mx/Medicion/Paginas/ PobrezaInicio.aspx

Coneval (2015). Hallazgos del estudio El acceso a los alimentos en los hogares: un estudio cualitativo, 2013-2014. Recuperado de: https:/www.coneval.org.mx/Evaluacion/ ECNCH/Documents/CIESAS_alimentacion.pdf

Coneval (2011a). Informe de evaluación de la politica de desarrollo social en México en materia de acceso a la alimentación. Recuperado de: https://www.coneval.org.mx/ Informes/Evaluacion/Documentos\%20tem\%C3\%A1 ticos\%20IEPDS2011/IEPDS\% 20_Alimentacion\%20_2011.pdf

Coneval (2011b). Evaluación de consistencia y resultados del Programa de Abasto Rural a cargo de Diconsa. Recuperado de: http://web.coneval.gob.mx/rw/resource/coneval/ eval_mon/1725.pdf

Coneval (2009). Informe de evolución histórica de la situación nutricional de la población y los programas de alimentación, nutrición y abasto en México. Recuperado de: https://www.coneval.org.mx/InformesPublicaciones/Paginas/Mosaicos/Informe-deEvolucion-historica-de-situacion-nutricional-de-la-población.aspx 
Cuéllar, J. (2011). Programa de Seguridad Alimentaria: experiencias en México y otros paises, D. F., México, CEPAL. Recuperado de: https://repositorio.cepal.org/bitstream/ handle/11362/26070/1/LCmexL1035_es.pdf

Diario Oficial de la Federación (DOF) (2013). Decreto por el que se establece el Sistema Nacional para la Cruzada contra el Hambre. Recuperado de: http://dof.gob. $\mathrm{mx} /$ nota_detalle.php?codigo $=5285363 \&$ fecha $=22 / 01 / 2013$

DOF (2019). Acuerdo por el que se emiten las Reglas de Operación de Prospera Programa de Inclusión Social, para el ejercicio fiscal 2019. Recuperado de: https://www.dof. gob.mx/nota_detalle.php? codigo $=5551581 \&$ fecha $=28 / 02 / 2019$

Franco, G. y Canela, F. (2016). Evolución de las políticas de desarrollo social en México: éxitos y fracasos. Revista Opera, 18, 159-181. Recuperado de: https://doi.org/10. 18601/16578651.n18.09

Hospital Infantil de México Federico Gómez (2009). Evaluación del diseño del Programa de Apoyo Alimentario a cargo de Diconsa, S. A. de C. $V$.

Huerta, M. (2005). El neoliberalismo y la conformación del Estado subsidiario. Política y Cultura, (24), 121-150. Recuperado de: http://www.scielo.org.mx/scielo.php?script= sci_arttext\&pid=S0188-77422005000200006

Huesca, L., López, R. y Palacios, M. (2016). El Programa de Apoyo Alimentario y la política social integral en la Cruzada contra el Hambre en México. Revista Mexicana de Ciencias Politicas y Sociales, 61(227), 379-408.

Instituto Nacional de Salud Pública (INSP). (2012). Programas de ayuda en México: cobertura y focalización. Recuperado de: http://ensanut.insp.mx/doctos/analiticos/ ProgAyudaAlimentaria.pdf

INSP (2015). Evaluación de Diseño Programa de Apoyo Alimentario.

López, R. y Sandoval, S. (2019). Propuestas de política para construir un proyecto nacional en materia de seguridad alimentaria. Los desafíos del nuevo gobierno. En: L. Huesca, G. Ordóñez y S. Sandoval (coordinadores), Los retos de la política social en el sexenio de la Cuarta Transformación . Tijuana, México: El Colegio de la Frontera Norte /Centro de Investigación en Alimentación y Desarrollo (en prensa).

MD CONSULTORÍA (2012). Diagnóstico de programas alimentarios de la SEDESOL. Recuperado de: https:/www.gob.mx/cms/uploads/attachment/file/31968/Diagnos tico_Alimentario_Ver\%0Asion_Completa_1_.pdf

Ordóñez, G. (2017). El Estado social en México: un siglo de reformas hacia un sistema de bienestar excluyente. Tijuana: Siglo XXI/ El Colegio de la Frontera.

Ordóñez, G. (2012). El régimen de bienestar en los gobiernos de la alternancia en México. Polis: Investigación y Análisis Sociopolitico y Psicosocial, 8(1), 213-240. 
Ordóñez, G. (2002). La política social y el combate a la pobreza en México. México: Universidad Nacional Autónoma de México.

Ordóñez, G. y Silva, A. (2019). Progresa-Oportunidades-Prospera: avatares, alcances y resultados de un programa paradigmático contra la pobreza. Papeles de Población, 25(99), 77-111.

Organización de la Naciones Unidas para la Alimentación y la Agricultura (FAO). (1996). Declaración de Roma sobre la Seguridad Alimentaria Mundial. Recuperado de: http://www.fao.org/docrep/003/w3613s/w3613s00.htm

FAO (2014). El Derecho a la Alimentación: compromisos pasados, obligaciones actuales, acciones para el futuro. Una retrospectiva de diez años de las Directices sobre el Derecho a la Alimentación. Recuperado de: http://www.fao.org/3/a-i4145s.pdf

Presidencia de la República (2008). Anuncia el Presidente Calderón acciones en apoyo a la economía familiar. Recuperado de: http://calderon.presidencia.gob.mx/2008/05/ anuncia-el-presidente-calderon-acciones-en-apoyo-a-la-economia-familiar/

Sánchez, S. (2018). AMLO disminuye en $28.5 \%$ el presupuesto para Agricultura y Desarrollo Rural. Expansión. Recuperado de: https:/expansion.mx/economia/ 2018/12/17/amlo-disminuye-presupuesto-agricultura-desarrollo-social

Secretaría de Agricultura y Desarrollo Rural (Sader) (2019a). Segalmex: productividad agroalimentaria y distribución de alimentos. Recuperado de: https://www.gob. $\mathrm{mx} /$ sader/es/articulos/segalmex-productividad-agroalimentaria-y-distribucion-dealimentos?idiom $=\mathrm{es}$

Sader (2019b). Una nueva canasta básica que asegura alimentación de calidad. Recuperado de: https:/www.gob.mx/sader/articulos/una-nueva-canasta-basica-que-asegura-alimen tacion-de-calidad?idiom $=$ es

Secretaría de Desarrollo Social (Sedesol) (2014). Acuerdo por el que se emiten las Reglas de Operación de Prospera, Programa de Inclusión Social, para el ejercicio fiscal 2015. Diario Oficial de la Federación, México.

Sedesol (2015). Lineamientos Especificos del Programa de Comedores Comunitarios para el Ejercicio Fiscal 2015. Sedesol, 1-27. Recuperado de: http:/www.gob.mx/cms/ uploads/attachment/file/29937/Lineamientos_Prog_Comedores_Comunitarios_2015 1_.pdf

Tamez, S., Ruiz, A., Ayala, C., y Ortiz, L. (2019). Programas de asistencia alimentaria e inseguridad alimentaria en los hogares mexicanos pobres con menores de edad. Estudios Sociales. Revista de Alimentación Contemporánea y Desarrollo Regional, 29(53). 
Yaschine, I., Ochoa, S. y Hernández, C. (2016). Cruzada Nacional Contra el Hambre: análisis de su diseño desde un enfoque de seguridad alimentaria. En: E. Valencia y G. Ordónez (Eds.), Nueva ronda de reformas estructurales en México, ¿nuevas politicas sociales? (pp. 422-461). Tijuana, México: El Colegio de la Frontera Norte . 